\title{
Korruption und die Ordnung der Geheimhaltung: zwischen ethischer Herausforderung und Doppelmoral ${ }^{* *}$
}

Dieser Artikel behandelt die charakteristische Geheimhaltungsstruktur von Korruption und den Schutz von Whistleblowern. Zentrale These ist, dass in der Korruptionsbekämpfung oftmals durch die Vorspiegelung von Moral und den Rekurs auf ein zu einseitiges Verständnis der Gerechtigkeit als eines ausgleichenden Prinzips im öffentlichen Raum, die sogenannte >Ordnung der Geheimhaltung`verstärkt und somit die Aufdeckung von Korruption gerade erschwert wird. Mit Bezugnahme auf den Begriff der >Billigkeit< als eines nicht auf Ausgleich abzielenden Begriffs der ethischen Gerechtigkeit wird eine neue Perspektive für die ethische Legitimation des Schutzes von Whistleblowern aufgezeigt, die zur Aufdeckung von Korruption beiträgt.

Schlagwörter: Korruption, Whistleblower, Geheimhaltung, Doppelmoral, Gerechtigkeit, Billigkeit

\section{Corruption and the Order of Secrecy: Between Ethical Challenges and Double Standards}

This paper discusses the protection of whistleblowers who break the >order of secrecy< that is characteristic for corruption. The central thesis is that in the fight against corruption, an often theatrical, public representation of morality and a one-sided but common understanding of justice as a balancing principle can strengthen this order of secrecy and may thus increase the difficulty of discovering cases of corruption. Instead, referring to sequity a as a different, non-balancing concept of ethical justice, the paper opens a new perspective on the ethical legitimization of the protection of whistleblowers.

Keywords: Corruption, Whistleblower, Secrecy, Double Standard, Justice, Equity

»[Bei korruptem Verhalten] gibt es keine offensichtlichen Opfer, die direkt spüren, dass ihnen Schaden zugefügt wurde» (Beck/Nagel 2012: 37).

\section{Einleitung}

Korruption bedeutet nicht nur den Austausch von Bestechungszahlungen gegen illegale Leistungen. Vielmehr bezeichnet der Begriff (von lat. corrumpere) in einem weiteren Sinn die Unterhöhlung öffentlicher Ordnung durch eine geheime Parallelordnung, eine sogenannte »Ordnung zweiter Art (Priddat/Schmid 2011),

* Dr. Verena Rauen, Institut für Wirtschaftsethik/Universität St.Gallen, Girtannerstrasse 8, CH-9000 St.Gallen, E-Mail: Verena.Rauen@unisg.ch, Forschungsschwerpunkte: Wirtschaftsphilosophie, Ethik, Zeit und Ökonomie, Korruption und Ungewissheit.

* Beitrag eingereicht am 30.06.2017; nach doppelt verdecktem Gutachterverfahren überarbeitete Fassung angenommen am 31.10.2017. 
innerhalb derer korrupte Akteure die ihnen anvertraute Macht zu privaten, d.h. nicht öffentlich legitimierten Zwecken, missbrauchen (vgl. Walzer 1983: 195ff.). Dabei spannt sich Korruption oftmals in einem ganzen Netzwerk korrupter Akteure auf, das sich als verdecktes System durch das persönliche Vertrauen (vgl. Boissevain 1974; Streck 1995; Baecker 2000; Lambsdorff/Teksoz 2002; Bluhm/ Fischer 2002; Hénaff 2010, 2011; Engels 2014) der Beteiligten sowie durch einen hohen Druck der Androhung interner Sanktionen bei Verrat der Netzwerke (vgl. Stenner 2011) schützt.

Deshalb wird im Folgenden die Frage im Vordergrund stehen, wie die `Ordnung der Geheimhaltung durch einen Begriff der Gerechtigkeit erodiert werden kann, der nicht auf dem Prinzip des Ausgleichs beruht: die durch Aristoteles eingeführte Konzeption der Billigkeit, die einen Ausgangspunkt für eine ethische Legitimierung des Schutzes von Whistleblowern bietet, denen für die Aufdeckung von Korruption eine besondere Rolle zukommt. Mit Rückgang auf die Moralkritik Friedrich Nietzsches, Emmanuel Levinas' und Marcel Hénaffs wird auf die Problematik der Doppelmoral eingegangen, die nicht hauptsächlich dadurch verursacht wird, dass moralische Prinzipien der Kommodifizierung anheim gestellt werden, wie es beispielsweise Walzer und Sandel nahelegen (vgl. Walzer 1983; Sandel 2012); vielmehr liegt eine Ursache der Doppelmoral und Verschleierung der Korruption in einer zu einseitigen Auffassung des Begriffs der Gerechtigkeit als eines Instruments der Allokation und Kompensation sowie in der individuellen Schuldzuweisung und oftmals theatralischen Repräsentation von Moral und Transparenz im öffentlichen Raum. Als Korrektiv der ausgleichenden Gerechtigkeit wird deshalb mit Rückgang auf Aristoteles' Nikomachische Ethik der Begriff der epieikeia oder Billigkeit als eine Form der ethischen Gerechtigkeit vorgestellt, die über die ausgleichende Gerechtigkeit hinausgehend und diese korrigierend - eine Grundlage für die ethische Legitimierung und weitreichende strukturelle Etablierung eines Schutzsystems für Whistleblower bietet.

Zur Korruptionsbekämpfung wurden in den vergangenen Jahrzehnten diverse Maßnahmen aus rechtlicher, ethischer und organisatorischer Sicht vorgestellt und etabliert. Dabei kristallisiert sich sowohl auf rechtlicher als auch auf unternehmensorganisatorischer Ebene das Problem heraus, dass die gängigen Verfahren der Korruptionsbekämpfung zwischen den beiden Polen der abschreckenden Wirkung hoher Strafen auf rechtlicher Basis (vgl. u.a. Yates 2015) und ComplianceSystemen einerseits ${ }^{1}$ und weichen $<$ Verfahren des Integritäts-Managements und der Etablierung von Whistleblowing-Systemen sowie von Systemen der Strafmilderung und der Anreizsetzung für Whistleblower andererseits oszillieren (vgl. Paine 1994; Lambsdorff/Nell 2007). Eine Zusammenführung dieser beiden Tendenzen der Korruptionsbekämpfung lässt sich in neueren Ansätzen des Compliance-Managements ausmachen, insofern diese compliance- und integritätsorientierte Konzepte kombinieren (vgl. Stessl 2012; Wieland 2005).

1 Siehe zur Zertifizierung von Compliance Management Systemen auch den Prüfungsstandard des Instituts der Wirtschaftsprüfer (IDW Prüfungsstandard 2011). 
Gerade angesichts der oben angesprochenen systemischen Vernetzung korrupter Akteure und der damit verbundenen Komplexität korrupter Strukturen innerhalb von Organisationen sind solche integrativen Ansätze sinnvoll. Betrachtet man jedoch die ethischen Grundlagen solcher Ansätze, so lassen diese oftmals ein Problem außer Acht, das in diesem Artikel im Vordergrund steht. Es geht dabei um die Doppelbödigkeit unternehmensethischer Maßnahmen der Korruptionsbekämpfung und -prävention im Hinblick auf die öffentliche Repräsentation von Moral sowie das grundlegende und in der Tradition der Moralkritik (vgl. u.a. Nietzsche (1999 [1887]); Levinas 2002, 1974; Waldenfels 2006) adressierte Missverhältnis von ethischen und ökonomischen Prinzipien sowie deren Anwendung auf den Begriff der Gerechtigkeit (vgl. Heimann 2015; Rauen 2015, 2016). Die integrative Anwendung von rechtlichen und ethischen Ansätzen der Korruptionsbekämpfung kann nur auf der Grundlage einer Rekalibrierung des Begriffs der Gerechtigkeit erfolgen, wie in diesem Aufsatz begründet werden soll.

\section{Die transparenten Schleier der Korruption}

Die Repräsentation von Unternehmensethik, Compliance und Transparenz im öffentlichen Raum ist kein Garant für ein effektives Vorgehen gegen oder eine Prävention von Korruption. Schließlich besteht das Problem der charakteristischen Geheimhaltungsstruktur von Korruption gerade angesichts der Einführung von Maßnahmen gegen eben diese: Insbesondere in der komplexen systemischen Struktur korrupter Netzwerke lässt sich eine verdeckte Ordnung der Regelumgehung ausmachen. In diesem Sinne wird die Wirksamkeit der gängigen Ansätze der Korruptionsbekämpfung, die in den vergangenen Jahren etabliert wurden (vgl. u.a. Yates 2015; IDW Prüfungsstandard 2011), in ein problematisches Licht gesetzt, da einhergehend mit dem Maß der Compliance-Vorschriften auch die Techniken ihrer Umgehung erweitert werden.

Die Repräsentation ethischer Maßnahmen im öffentlichen Raum kann mithin dazu beitragen, Korruption innerhalb von Organisationen zu befördern, indem ihre charakteristische Geheimhaltungsstruktur zementiert wird (vgl. Boissevain 1974; Streck 1995; Hénaff 2011). So beschreiben die Anthropologen Jeremy Boissevain und, mit Bezug auf seine Arbeiten zur anthropologischen Dimension der Korruption, Bernhard Streck die Vorspiegelung von Moral im öffentlichen Raum als tendenziell korruptionsfördernd, insofern diese der Verschleierung von Korruption dienlich sein kann.

»Boissevain verglich die gesellschaftlichen Normen mit Vorhängen, die man zuzieht, um sich dahinter ungenierlich benehmen zu können. Diese Vorhänge scheinen nun im Verlauf der überschaubaren Kulturgeschichte immer dichter gewebt worden zu sein, da das elementare Nehmen und Geben aus nackter Profitgier durch den >Prozeß der Zivilisation< auf immer deutlichere Mißbilligung stieß. (...) In Boissevains Bild ist der kulturelle Vorhang fadenscheinig geworden. Man kann hindurchsehen und erblickt auch an der Schwelle zum dritten Jahrtausend: homo manipulator, den Urmenschen, bei der immer noch eigennützigen Pflege seiner Beziehungen « (Streck 1995: 5). 
Gerade der in der Korruptionsbekämpfung prominente Begriff der Transparenz erhält angesichts der Ausführungen Boissevains und Strecks einen ambivalenten Charakter: Denn Transparenz dient nicht nur der >Durchsichtigkeit $<$ und damit der Aufklärung (vgl. Foucault 1977; Alloa 2017), sondern kann durch die Erhöhung der Komplexität von Informationen sowie durch die Täuschungseffekte einer doppelbödigen öffentlichen Repräsentation von Anti-Korruptionsmaßnahmen einen Effekt der Verschleierung erzeugen, der umso wirksamer ist, je mehr er auf die Dimension der Moral verweist:

»Transparent aber sind, wie wir gesehen haben, die Vorhänge geworden, mit denen die Moderne ihre archaischen Anteile zuhängen wollte« (Streck 1995: 7).

Aus ethischer Perspektive lässt sich das Problem der Repräsentation von Moral nicht dadurch lösen, dass zwischen der 'falschen und der >richtigen` Moral wie zwischen den beiden Ebenen eines doppelten Bodens unterschieden wird. Vielmehr wird im Folgenden das Problem im Vordergrund stehen, dass ein zu einseitiges Verständnis der Moral auf der Grundlage eines auf dem Prinzip des Ausgleichs basierenden Gerechtigkeitsbegriff sowie eine daraus resultierende Verwechslung der Konzeption der Strafgerechtigkeit mit ethischer Gerechtigkeit (vgl. Cohen 1904) dazu beitragen, die Eindämmung von Korruption zu blockieren. Entgegen einer Betonung der Individualisierung der Schuld und der Übernahme von >Verantwortung Einzelner im Falle der Aufdeckung von Korruption, soll in diesem Aufsatz ein Konzept der Ethik im Sinne einer kritischen Reflexion und Enthaltung von Schuldurteilen als Gegengewicht zu Tendenzen der Strafgerechtigkeit in die Waagschale geworfen werden. Dabei ist es dezidiert nicht das Ziel, die Strafgerechtigkeit zu Gunsten ’weicher< Verfahren der Anti-Korruptionsmaßnahmen auszusetzen. Vielmehr ist es das Anliegen dieses Artikels, einen angemessenen Konterpart zur Strafgerechtigkeit durch diejenigen ethischen Konzepte bereitzustellen, die die Strafgerechtigkeit und die damit verbundenen Praktiken der öffentlichen Schuldzuweisung und Verurteilung reflektieren und im Einzelfall aussetzen können, insbesondere dann, wenn die Aussetzung der Strafgerechtigkeit im Einzelfall zur Freisetzung weitreichender Effekte der Korruptionsbekämpfung führen kann, wie dies beispielsweise im Falle der Strafmilderung oder gänzlicher Amnestie für Whistleblower der Fall sein kann. So fordert der Whistleblower >John Doe`, der 2015 der Süddeutschen Zeitung anonym die sogenannten >Panama Papers zuspielte, einen weitreichenden Schutz für Whistleblower und kritisiert die immer noch häufig vorkommende juristische Verfolgung von Whistleblowern (vgl. dazu auch Fischer-Lescano 2015) im internationalen Recht:

"Ich habe mitangesehen, was mit Whistleblowern und Aktivisten in den USA und Europa geschehen ist, wie ihr Leben zerstört wurde, nachdem sie Vorgänge öffentlich gemacht hatten, die offensichtlich kriminell waren. Edward Snowden sitzt in Moskau fest, im Exil, weil die Obama-Regierung auf Grundlage des Anti-Spionage-Gesetzes Haftbefehl gegen ihn erlassen hat. (...) Bradley Birkenfeld bekam von der US-Steuerbehörde Millionen für seine Informationen über die Schweizer Bank UBS - und wurde dennoch vom US-Justizministerium verurteilt. Antoine Deltour wird momentan in Luxemburg der Prozess gemacht, weil er Journalisten Informationen zur Verfügung gestellt hat, die offenlegten, wie das Land mit enormen Steuervergünstigungen internationale Firmen angelockt hat, und damit letztlich seine europäischen Nachbarländer um Milliarden an Steuergeldern gebracht hat « (Doe 2016: 1). 
Angesichts der Wichtigkeit der Aufdeckung von Korruption durch Whistleblower fordert `John Doe` deren >Immunität vor dem Gesetz` und macht damit auf die Wichtigkeit einer Rekalibrierung des Begriffs der ausgleichenden Gerechtigkeit für die Korruptionsbekämpfung aufmerksam:

»Whistleblower, die das Richtige tun, indem sie Straftaten aufdecken, egal ob als Insider oder Outsider, verdienen Immunität vor dem Gesetz. Punkt. Solange Regierungen keinen Rechtsschutz für Whistleblower garantieren, sind Strafverfolgungsbehörden weiterhin abhängig von ihren eigenen Informationsquellen oder von medialer Berichterstattung, um an entsprechende Dokumente zu gelangen« (ebd.).

Im Folgenden wird es um die Frage gehen, inwiefern ein Verständnis der Ethik, das über ein auf ökonomischen Prinzipien beruhendes Konzept der Gerechtigkeit hinausgeht, zur Aufdeckung und Prävention von Korruption beitragen und somit die vorhandenen Effekte der rechtlichen Bestrafung von Korruption überhaupt erst freisetzen kann. Im Spannungsfeld von Individual- und Systemethik wird als Korrektiv der Strafgerechtigkeit die von Aristoteles eingeführte und von Hermann Cohen und Emmanuel Levinas weiter ausgearbeitete Konzeption der epieikeia oder Billigkeit im Vordergrund stehen.

\section{Das Dilemma der Korruptionsaufdeckung}

Weitreichende Effekte der Strafgerechtigkeit und der rechtlichen Sanktionierung von Korruption können erst dann freigesetzt werden, wenn Korruption überhaupt zur Aufdeckung kommt. Dabei kommt Whistleblowern und der gezielten Anreizsetzung für Whistleblower durch eine In-Aussicht-Stellung von Straferlass eine entscheidende Bedeutung zu (vgl. Rose-Ackermann 1999: 53ff.). Wie das oben angeführte Zitat des Whistleblowers `John Doer veranschaulicht, wird die Aufdeckung von Korruption jedoch oftmals durch das Rechtssystem erschwert, insofern Hinweisgeber mit massiven rechtlichen und sozialen Sanktionen rechnen müssen, wenn es ihnen nicht gelingt, anonym zu bleiben. So attestiert ein juristisches Kurzgutachten von 2015 das Fehlen eines "systematischen, kohärenten und umfassenden Schutzsystem(s) « (Fischer-Lescano 2015: 9) für Whistleblower im deutschen Rechtssystem:

»Whistleblowerinnen und Whistleblower sind existenziellen Gefahren - drohender Verlust des Arbeitsplatzes, disziplinarrechtliche Maßnahmen, strafrechtliche Verfolgung, außerrechtliche Sanktionen - ausgesetzt, die sie ggf. davon abhalten können, ihre für die demokratische Öffentlichkeit wichtige Funktion auszuüben. Für die Beschuldigten birgt das Whistleblowing existenzielle - wirtschaftliche, politische, strafrechtliche - Risiken und die Öffentlichkeit ist in ihrer Kontrollmöglichkeit eingeschränkt, was wiederum Auswirkungen für die Gewährleistung der Güter im Wohlfahrtsstaat - Sicherheit, Gesundheit, Umweltschutz, Menschenrechte - hat « (ebd.: 3).

Wenngleich die Anwendung von Strafgerechtigkeit in bereits aufgedeckten Fällen der Korruption in ihrer Wirksamkeit nicht bezweifelt wird, wirft die Tatsache, dass ein ausreichender rechtlicher Schutz für Whistleblower nach wie vor nicht etabliert ist, die Frage nach einer angemessenen Ausbalancierung des Gerechtigkeitsbegriffs auf. Die Einführung eines Anreizsystems, das potenziellen Hinweisgebern eine Motivation zur Aufdeckung von Korruption gibt, um daraufhin über- 
haupt erst mit rechtlichen Sanktionen gegen Korruption vorgehen zu können, würde durch eine solche Rekalibrierung eine weitere ethische Legitimation erhalten.

Auf der Grundlage spieltheoretischer Überlegungen wurde ein System sogenannter sasymmetrischer Strafen blower bereits 2007 von Lambsdorff und Nell vorgeschlagen. Ein solches System der Anreizsetzung eröffnete an entscheidenden Stellen einer korrupten Transaktion, beispielsweise nach dem Angebot oder der Übergabe eines Bestechungsgeldes, Ausstiegsmöglichkeiten für potentielle Hinweisgeber, indem diesen Straferlass oder gezielte Anreize wie Prämien in Aussicht gestellt würden; dabei würden die korrupten Interaktionspartner durch asymmetrische Strafen, d.h. Straferlass für denjenigen, der den korrupten Deal zur Aufdeckung bringt, und volle Bestrafung für denjenigen Beteiligten, der >verraten $<$ wurde, systematisch gegeneinander ausgespielt:

»We propose the following asymmetric design: expected criminal sanctions for accepting bribes should be low and those for illicitly supplying favorable treatment (e.g. awarding a public contract) to the briber high; in turn, expected penalties for paying bribes should be severe, while those for accepting the (illicit) favorable treatment mild. Moreover, we show that a bribe-taker shall only be granted ex-ante leniency as long as he does not reciprocate a bribe, whereas a bribe-giver shall be entitled to leniency only if he self-reports after having received the favorable treatment by the bribe-taker " (Lambsdorff/Nell 2007: 4).

Im Rahmen der Prinzipal-Agent-Klienten-Theorie wird Korruption somit durch ein Gefangenendilemma vorgestellt, innerhalb dessen die korrupten Partner in Ungewissheit darüber verbleiben, ob der jeweils andere den korrupten Deal verraten wird (vgl. ebd.: 2f.). Die grundsätzliche Einsicht, dass der korrupte Deal der Geheimhaltung unterliegt und somit nicht durch offiziell legitimierte Verträge abgesichert werden kann, wird dabei zum Ansatzpunkt genommen, um gegen Korruption vorzugehen. Mit dem Ziel einer Erhöhung der Ungewissheit innerhalb korrupter Netzwerke wird durch ein System asymmetrischer Strafen die Kosten-/ Nutzen-Erwartung der korrupten Akteure ins Ungleichgewicht gebracht: unzuverlässige korrupte Deals sind teurer, da sie ein hohes Risiko der Aufdeckung bergen (vgl. Priddat/Schmid 2011; Beck/Nagel 2012). Dadurch würde ein sinnvoll etabliertes System der Strafmilderung einerseits präventiv wirken, da das erwartete Risiko möglicherweise eine Entscheidung gegen Korruption hervorrufen könnte. Andererseits würden die Effekte der abschreckenden Wirkung der Sanktionierung überhaupt erst in Kraft gesetzt, insofern es durch ein System asymmetrischer Bestrafung zur Aufdeckung der Korruption käme. Das System einer asymmetrischen Bestrafung erforderte dabei vor allem einen umfassenden Schutz von Hinweisgebern bzw. eine klare Erwartung (»ex ante«; vgl. Lambsdorff/Nell 2007: 1) der umfassenden Strafmilderung für Whistleblower, insofern diese die Aufdeckung von Korruption ermöglichen. Jedoch ist ein systematisch etablierter Schutz für Whistleblower in den meisten Rechtssystemen nicht gegeben, auch wenn punktuell Strafmilderung in Aussicht gestellt wird:

»Because of its potential to shatter corrupt actors' trust in reciprocity and in mutual silence, an asymmetric design of sanctions, coupled with strategically granting leniency, might unleash higher deterrent effects of anti-corruption legislation (...). Yet, in most 
countries (...) ex-ante leniency is the exception rather than the rule (Lambsdorff/Nell 2007: 12).

Asymmetrische Bestrafung und Straferlass konfligieren mit der verbreiteten Erwartung der Ausübung ausgleichender Gerechtigkeit und der damit einhergehenden Autorität der Strafgerechtigkeit. So gibt beispielsweise das 2015 veröffentlichte Memorandum von US Deputy Attorney Sally Q. Yates ein deutliches Exempel der Akzentuierung der abschreckenden Wirkung individualisierter Strafgerechtigkeit für die internationale Korruptionsbekämpfung (vgl. Yates 2015; Emmenegger 2016). Yates schreibt die besonders hohe Bestrafung von individuellen Verantwortlichen, insbesondere von verantwortlichen Topmanagern von Unternehmen, auf die Agenda der Korruptionsbekämpfung und macht diese zur Voraussetzung für einen Vergleich mit einem Unternehmen. In diesem Sinne fordert das Memorandum explizit die »Individual Accountability for Corporate Wrongdoing " (Yates 2015) und damit einhergehend die Herausgabe von Informationen zu individuellen Verantwortlichen, die Korruption im Unternehmen unterstützt haben, durch das Unternehmen.

Mit Yates' Memorandum wird dem allgemein etablierten Verständnis ausgleichender Gerechtigkeit genüge getan, indem die Verantwortung für Fälle der Korruption nicht auf die betroffene Organisation abgewälzt, sondern individualisiert wird. Jedoch wurden bereits kurz nach der Veröffentlichung des Memorandums Stimmen laut, die eine Erschwerung der Aufdeckung von Korruption durch die Individualisierung der Strafen verlauten lassen (vgl. Emmenegger 2016: 1054f.). So wird der spärliche Informationsfluss bei der Untersuchung von Korruptionsfällen kritisiert, der darauf zurückgeführt werden könnte, dass Unternehmen zur Erreichung eines Vergleichs gezwungen werden, individuelle Mitarbeiter zu beschuldigen und auszuliefern, obwohl die Aussicht auf das tatsächliche Zustandekommen des Vergleichs für das Unternehmen als Ziel dieser Prozedur oftmals ungewiss ist (vgl. ebd.: 1055). Zudem wird die Motivation potenzieller Whistleblower deutlich abgeschwächt, Korruption zur Aufdeckung zu bringen, da eine hohe Wahrscheinlichkeit der individuellen Bestrafung gegeben ist.

Obwohl die Wirkung der Bestrafung von Korruption nicht als solche in Frage steht, verfehlt das Vorgehen von Yates die durch Lambsdorff und Nell adressierte Problematik der Korruption, die in deren Verborgenheit liegt. Die Individualisierung der Bestrafung erscheint wenig effektiv im Hinblick auf eine nachhaltige Bekämpfung von Korruption, insofern diese durch ein komplexes Netzwerk korrupter Akteure und durch Geheimhaltung gekennzeichnet ist. Durch die Androhung und Verhängung hoher Strafen und eine damit einhergehende Betonung der Strafgerechtigkeit wird die Praxis der Geheimhaltung im Gegenteil weiter motiviert.

\section{Das Konzept der Korruption zwischen Individual- und Systemethik}

Entscheidend für eine Betrachtung der oben genannten Polarität zwischen der abschreckenden Wirkung der Strafgerechtigkeit und der Etablierung von Strafmil- 
derung für Whistleblowing aus ethischer Perspektive ist eine angemessene Berücksichtigung der Spannung zwischen Individual- und Systemethik.

Sowohl das Modell der asymmetrischen Strafen als auch die Akzentuierung abschreckender Strafgerechtigkeit betrachten Korruption individualisiert als die Machenschaft einzelner Straftäter und Mitwisser, die entweder durch Bestrafung abgeschreckt oder durch Strafmilderung zur Aufdeckung von Korruption motiviert werden sollen. Sie folgen damit gängigen Definitionen von Korruption, wie etwa der von Transparency International: Korruption als Missbrauch anvertrauter Macht zu privaten Zwecken; bzw. in verbreiteten Ansätzen der Ökonomik und den Sozialwissenschaften: Korruption als geheimer Deal zwischen Agent und Klient zu Lasten des Prinzipals im Rahmen des Prinzipal-Agent-Klienten Modells (vgl. Klitgaard 1991: 223; Homann 1997: 192), als »unmoralischer Tausch" (Neckel 1995: 9), als »sittenwidrige Käuflichkeit « (Wieland 2005: 43); oder in der Moralphilosophie: als Tausch unveräußerlicher moralischer Normen gegen ökonomische Preise (vgl. Sandel 2012: 46). Korruption ist insbesondere von der institutionellen Rahmenordnung abhängig und setzt oftmals als breit angelegtes Netzwerk an deren systemischen Schwachstellen an (vgl. Beck/Nagel 2012; Stenner 2011; Schmid 2011; Priddat 2005), so dass eine individualisierte bzw. auf individuelles Fehlverhalten bezogene Definition der Korruption zu kurz greift, um diesem Phänomen gerecht zu werden (vgl. Lambsdorff/Nell 2007; Luhmann 1997: 837, 929). Basierend auf der Netzwerktheorie schlägt Wieland eine Erweiterung der von Klitgaard (1991) vorgestellten Prinzipal-Agent-Klienten-Theorie der Korruption vor, indem er von einer doppelten Prinzipalstruktur ausgeht und den Prinzipal sowohl auf Seiten des Auftragnehmers als auch auf der des Auftraggebers verortet (vgl. Wieland 2005: 49f.). Zur Bekämpfung der Korruption schlägt er eine Kombination von Compliance- und Integritätsmaßnahmen für die Etablierung umfassender »Wertemanagementsysteme « (ebd. 52ff.) innerhalb der Governancestrukturen von Institutionen vor. Auch Beck/Nagel (2012) sowie Stenner (2011) argumentieren damit, dass Korruption sich als Netzwerkphänomen in erster Linie in sschlechten< Institutionen mit misslingenden Ordnungsstrukturen abspielt. Insbesondere die Forschungen zur Korruption aus interkultureller Perspektive, wie etwa zur Korruption im nigerianischen Warlord-System (vgl. Schmid 2011) oder zur korrupten Struktur der Yakuza in Japan (vgl. Stenner 2011), weisen, auch wenn sie völlig unterschiedliche kulturelle und politische Gegebenheiten betreffen, gemeinsam auf das Problem hin, dass korrupte Netzwerke oftmals an systemische Schwachstellen politischer Rahmenordnungen anknüpfen und eine durch öffentliche Strukturen nicht ausreichend ausgeübte oder geschützte Funktion übernehmen. Dies betrifft bei den nigerianischen Warlord-Strukturen etwa die scheinbare 'Sicherung von territorialen Besitz- und Herrschaftsansprüchen (vgl. Schmid 2011) angesichts instabiler politischer Verhältnisse, innerhalb derer Eigentumsrechte nicht ausreichend gesichert sind. Im Beispiel der Yakuza betrifft die systemische Funktion die Aufrechterhaltung vordergründiger Sicherheit durch eine Unterdrückung von Kleinkriminalität in den von ihr kontrollierten Bereichen wie etwa des Rotlichtmilieus, aber auch die Beschleunigung administrativer Vorgänge in zivilrechtlichen Angelegenheiten durch Bestechungen (vgl. Stenner 2011). 
Es lässt sich an diesem Punkt nicht nur eine Erweiterung und Umdefinierung der klassischen individualisierten Definition von Korruption zu Gunsten von systemischen Netzwerkphänomenen vornehmen, sondern auch eine kritische Hinterfragung der Prinzipal-Agent-Klienten-Theorie (vgl. Klitgaard 1991; Wieland 2005) zur Erklärung von Korruption: Im klassischen Modell wurde Korruption als ein geheimer Deal zwischen dem Agenten (Angestellter, Beamter) und dem Klienten (Kunde, potenzieller Auftragnehmer) aufgefasst, der zu einem Bruch des bestehenden Vertrags sowie zu einer Informationsasymmetrie zwischen Agent und Prinzipal führt (vgl. Klitgaard 1991; Homann 1997; Beck/Nagel 2012). Priddat und Schmid (2011) beschreiben diesen Vorgang der Bildung einer durch die Informationsasymmetrie entstehenden Parallelordnung neben der öffentlichen Ordnung als die bereits angeführte "Ordnung zweiter Art ", in deren Rahmen sich das korrupte Verhältnis zwischen den Beteiligten wie dasjenige eines Parasiten zu seinem Wirt, dessen Funktion in diesem Fall durch den Prinzipal übernommen wird, realisiert.

Unter Einbeziehung der oben angesprochenen Verhältnisse der systemischen Korruption muss diese Perspektive jedoch verschoben werden. Es liegt nahe zu fragen, inwiefern der Prinzipal selbst in das Netzwerk der Korruption verstrickt ist, wie es beispielsweise Wieland in seiner Erweiterung der Prinzipal-Agent-Klienten-Theorie durch die Einbeziehung des Prinzipals in das Netzwerk der Korruption nahelegt (vgl. Wieland 2005). Priddat (2005) führt diesen Aspekt ebenfalls im Rahmen seiner Überlegungen zur Ordnung zweiter Art anhand der negativen Auswirkungen der Public-Private Partnership sowie des Lobbyismus, im Sinne einer Begünstigung korrupter Beziehungen durch die Nähe zwischen Privatwirtschaft und Politik, aus.

Aus moralphilosophischer Sicht ist die Verstrickung des Prinzipals in die Netzwerke der Korruption ebenfalls reflektiert worden, insbesondere durch Michael Walzer, der als Kriterium der Korruption nicht lediglich einen geheimen Deal zwischen Agent und Klient ansetzt, sondern jegliche politische Rahmenordnung für ursächlich hält, innerhalb derer ein zu starker Übergriff des Markts auf politische Machtbereiche und insbesondere auf soziale Sphären wie beispielsweise das Bildungs- oder Gesundheitswesen erfolgt (vgl. u.a. Walzer 1983: 50). Auf der Grundlage einer öffentlichen Legitimierung stellt die Blockierung von ökonomischen Tauschprozessen, die für Walzer einen Übergriff auf gesellschaftlich zu schützende soziale Sphären bedeuten würde, einen wichtigen Aspekt der Korruptionsbekämpfung dar (vgl. ebd.: 156ff.). Die oben angebrachten Beispiele der Yakuza und Warlord-Systeme unterlägen im Sinne Walzers immer noch der Ordnung der Geheimhaltung, auch wenn sie in der jeweiligen Gesellschaft allgegenwärtig präsent sind. Denn die systemische Funktion, die die Korruption in den jeweiligen politischen Ordnungen einnimmt, ist nicht durch öffentliche Gesetzgebung und einen demokratischen Entscheidungsprozess legitimiert.

Problematisch ist dennoch die von Michael Sandel 1998 im Anschluss an Walzer ausgeführte Definition der Korruption, die er durch einen 'Mangel an Moral verursacht sieht und damit wieder auf die Ebene individueller Verfehlung zurückführt. Denn Korruption wird von Sandel als das moralische Fehlverhalten von Individuen vorgestellt, indem diese moralische Normen einer ökonomischen Tau- 
schlogik preisgeben und in diesem Zug eigentlich unveräußerliche Werte gegen ökonomische Preise eintauschen (vgl. Sandel 2012: 46, 94). Zwar setzt der ursprünglich von Walzer vorgebrachte Ansatz zur Eindämmung von Korruption an einer systemischen Stelle an, indem er durch die Regulierung und >Blockierung von Tauschvorgängen, die seiner Ansicht nach unmoralisch sind, einen Schutz vor Korruption schaffen will. Jedoch ist die Weiterführung eines solchen Korruptionskonzepts durch Sandel, die darin besteht, dass dieser zwischen Korruption und ökonomischem Zwang (Coercion) unterscheidet (vgl. ebd.: 94ff.), klar ein Rückschritt im Hinblick auf eine differenzierte Betrachtung des Korruptionsphänomens als einer systemischen Netzwerkstruktur.

Sandel differenziert zwischen dem Zwang, der Individuen aus einer ökonomischen Notlage heraus dazu bringt, ’korrupte< Tauschvorgänge zu vollziehen, wie beispielsweise den Verkauf eines Organs zu tätigen, und der Korruption, die in einer Missachtung und Veräußerung der von ihm und Walzer angesetzten moralischen Werte liegt (vgl. ebd.). So sei dem Problem des 'Zwangs durch faire Rahmenbedingungen, d.h. eine bessere Regulierung von Tauschvorgängen und eine gerechtere Allokation von Gütern zu begegnen, während Korruption im Sinne einer Veräußerung moralischer Werte sowohl unter fairen als auch unter unfairen ökonomischen und politischen Rahmenbedingungen stattfinden könne (vgl. ebd.: 95). Deshalb wird Korruption für Sandel durch ein Übergreifen der Marktlogik auf die Sphäre der Moral verursacht und besteht in einer Veräußerung moralischer Normen zu ökonomischen Preisen. Korruption vollzieht sich demzufolge durch einen individuellen oder auch kollektiven >Mangek an Moral und nicht in erster Linie durch eine unzureichende gesetzliche oder institutionelle Rahmenstruktur, wie es beim Phänomen des Zwangs der Fall ist:

»We often associate corruption with illicit payoffs to public officials. But (...) corruption also has a broader meaning: we corrupt a good, an activity, or a social practice whenever we treat it according to a lower norm than is appropriate to it " (Sandel 2012: 46).

Davon abgesehen, dass die moralischen Normen, sozialen `Sphären gen Phänomene, die Walzer (1983: 157ff.) und Sandel (2012: 9ff.) in ihren Arbeiten als vor der Marktlogik schützenswert präsentieren, einer recht beliebigen Katalogisierung unterliegen, so dass kaum ausgewiesen ist, welches inhaltliche Kriterium über eine einfache Mehrheitsentscheidung hinausgehend für die Festsetzung und öffentliche Legitimierung eines moralischen Standards oder einer zu schützenden sozialen Sphäre gelten sollte, erscheint die Betrachtung der Moral, die die genannten kommunitaristischen Ansätze bieten, reduktionistisch (vgl. zur Kritik an Walzer und Sandels Ansatz im Hinblick auf Korruption: Hénaff 2010: 397ff.; Rauen 2016: 112).

Denn es sind nicht einfach nur die richtige Anwendung und öffentliche Ausarbeitung moralischer Normen sowie der Schutz des sogenannten >Guten', den beide Autoren propagieren (ohne dabei überzeugend auszuweisen, wie in einer pluralistischen Gesellschaft das >Gute überhaupt positiv definiert werden soll), die angesichts von Korruption und deren Charakteristik der Verborgenheit und Geheimhaltung in Frage stehen. Vielmehr betreffen die angesprochenen Probleme der Verschleierung und Doppelmoral sowie der systemischen Netzwerkstruktur 
der Korruption die Notwendigkeit der Reflexion und Kritik der Moral und des Konzepts ethischer Gerechtigkeit selbst.

\section{Moral als Instrument der Doppelmoral}

Moral ist nicht per se sgut . Sie bedarf einer gründlichen Reflexion ihrer Entstehungsgeschichte und konzeptuellen Prämissen, insbesondere im Hinblick auf ihre seit der Antike belegbare Verbindung mit politischen Machtstrukturen und ökonomischen Prinzipien. Entsprechend einer gängigen Auffassung in der Sozialphilosophie wird im Folgenden zwischen Ethik im Sinne einer theoretischen Ebene, auf der über moralische Normen reflektiert wird, und Moral im Sinne eines gesellschaftlich und kulturell tradierten Normen- und Regelkatalogs unterschieden (vgl. Levinas 2002; Waldenfels 2006). Die öffentliche Repräsentation von Moral im Hinblick auf die Bekämpfung und Prävention von Korruption wird insbesondere durch das Compliance Management sowie durch die öffentliche Repräsentation von Unternehmensethik abgedeckt. Dabei gewinnt für das Compliance Management bereits seit den neunziger Jahren (vgl. Paine 1994) neben der Einhaltung von Regeln und Normen sowie Systemen der Sanktionierung von Regelverstößen auch die Reflexion und Entwicklung eines Integritätsmanagements und damit einhergehend einer entsprechenden Unternehmenskultur an Stellenwert (vgl. Stessl 2012; Wieland 2005). Problematisch ist, dass selbst unter Einbeziehung des Begriffs der Integrität in Compliance Management-Systeme ein kritischer Blick auf den Stellenwert der Moral für Korruption oftmals fehlt.

Denn zentrale moralische Begriffe, wie zum Beispiel Gerechtigkeit oder Verantwortung, werden oftmals zu einseitig, ohne dass dies jedoch explizit reflektiert würde, auf Prinzipien des Schuldenausgleichs oder der Distribution von Gütern zurückgeführt (vgl. Heidbrink/Rauen 2016: 191ff.), die eine große Nähe zu ökonomischen Prinzipien aufweisen. Eine derartig reduktionistische Repräsentation von Moral ist mit ursächlich für die beizeiten hypokritisch anmutende Praxis der Schuldzuweisung und der Forderung nach einer >Übernahme von Verantwortung durch bestimmte Individuen im öffentlichen Diskurs (vgl. u.a. Lübbe 2001). Derartig ambivalente Szenen wie der Rücktritt Winterkorns im VW-Dieselskandal mit den Worten: "Als Vorstandsvorsitzender übernehme ich die Verantwortung für die bekannt gewordenen Unregelmäßigkeiten bei Dieselmotoren und habe daher den Aufsichtsrat gebeten, mit mir eine Vereinbarung zur Beendigung meiner Funktion als Vorstandsvorsitzender des Volkswagen-Konzerns zu treffen. Ich tue dies im Interesse des Unternehmens, obwohl ich mir keines Fehlverhaltens bewusst bin « (Die Welt: 23.09.2015), sowie das Auftreten extremer Diskrepanzen zwischen Außenrepräsentation von Unternehmensethik und unternehmensinternen Missständen nach dem Bekanntwerden von Korruptionsfällen, wie im Falle Siemens (vgl. Wolf 2009), stellen im öffentlichen Raum keine Seltenheit dar.

Die ambivalente Rolle der Moral als Instrument der Doppelmoral im Hinblick auf das Problem der Korruption liegt jedoch nicht einfach in einem sfalschen Gebrauch oder Missbrauch der Moral, wie es etwa Walzer (1983) oder Sandel 
(2009) nahe legen. Vielmehr besteht die Anfälligkeit der Moral für Doppelmoral in der zu einseitigen Betonung der ökonomischen Dimension der Moral, insbesondere gestützt durch ein auf Prinzipien des Ausgleichs und der Distribution basierendes Verständnis der Gerechtigkeit sowie die gängige Praxis der Zuweisung individueller Schuld, wie insbesondere Nietzsche in seiner Moralkritik hervorhebt:

»Haben sich diese bisherigen Genealogen der Moral auch nur von Ferne Etwas davon träumen lassen, dass zum Beispiel jener moralische Hauptbegriff 'Schuld seine Herkunft aus dem sehr materiellen Begriff ,Schulden Vergeltung sich vollkommen abseits von jeder Voraussetzung über Freiheit oder Unfreiheit des Willens entwickelt hat? « (Nietzsche 1999 [1887]: 297)

Eine moralkritische Dimension der Gerechtigkeitskonzeption findet sich insbesondere in Nietzsches oben zitierter Genealogie der Moral, in der dieser den Gerechtigkeitsbegriff durch eine systematische Verwechslung zwischen ökonomischen Schulden und moralischer Schuld determiniert sieht. Unter der zu einseitigen Maßgabe der Abzahlung und des Ausgleichs von Schuld, wird Gerechtigkeit für Nietzsche somit zu einem moralischen Machtmittel:

$»(. .$.$) )jedes Ding hat seinen Preis; Alles kann abgezahlt werden <-$ dem ältesten und naivsten Moral-Kanon der Gerechtigkeit, dem Anfange aller >Gutmüthigkeit‘, aller >Billigkeit‘, alles sguten Willens‘, aller >Objektivität` auf Erden. Gerechtigkeit auf dieser ersten Stufe ist der gute Wille unter ungefähr Gleichmächtigen, sich miteinander abzufinden, sich durch einen Ausgleich wieder zu 'verständigen - - und, in Bezug auf weniger Mächtige, diese unter sich zu einem Ausgleich zu zwingen « (ebd.: 306f.).

Jedoch lässt sich im Rahmen einer ethischen Reflexion und Kritik der Moral ein Gegengewicht zu Nietzsches Akzentuierung des auf ökonomischen Prinzipien beruhenden Begriffs der Gerechtigkeit ausmachen, der auf Aristoteles' Nikomachische Ethik (vgl. Aristoteles 2006) zurückgeht. So stellt Aristoteles im fünften Buch der Nikomachischen Ethik seinen Ausführungen zur ausgleichenden und distributiven Gerechtigkeit die sogenannte Billigkeit oder epieikeia als Korrektiv bei, die zur Untermauerung der Idee des Systems der asymmetrischen Bestrafung und der damit einhergehenden Weiterentwicklung des Umgangs mit Whistleblowern dienen könnte.

\section{Gerechtigkeit: dies- und jenseits ökonomischer Bedingungen}

Die Relevanz ausgleichender Gerechtigkeit und der davon abgeleiteten Konzeption der retributiven Gerechtigkeit steht in ihrer Bedeutung für die Korruptionsbekämpfung nicht in Frage. Jedoch ist die ausgleichende Gerechtigkeit aus ethischer Perspektive nicht die einzige Form der Gerechtigkeit, die zur Bekämpfung und Prävention von Korruption relevant ist. Vielmehr bietet Aristoteles ein Korrektiv zur ausgleichenden Gerechtigkeit, das für den Ansatz der asymmetrischen Bestrafung eine ethische Legitimation bietet.

In der Nikomachischen Ethik hebt Aristoteles die Relevanz der sogenannten Billigkeit, epieikeia, als Korrektiv zur ausgleichenden Gerechtigkeit hervor (vgl. Aristoteles 2006: 188ff., 1137b-1138a). Dabei erhält die Billigkeit als ein Verzicht auf die Ausübung der ausgleichenden Gerechtigkeit in solchen Fällen ethische Rele- 
vanz, in denen die Anwendung des strikten Rechts und der Strafgerechtigkeit zu weiterer Ungerechtigkeit führen könnte (vgl. Rauen 2015: 102ff.). Sie stellt somit eine besondere Form der Gerechtigkeit dar, die in ihrer ethischen Relevanz sogar über die ausgleichende Gerechtigkeit hinausgeht:

»Denn das Billige ist, obwohl es besser ist als eine bestimmte Art des Gerechten, gerecht, und es ist nicht in der Weise besser als das Gerechte, als wenn es zu einer anderen Gattung gehörte. Gerechtes und Billiges ist also dasselbe, und während beide gut sind, ist das Billige das Überlegene« (Aristoteles 2006: 188, 1137b).

Die ethische Überlegenheit der Billigkeit gegenüber der ausgleichenden Gerechtigkeit bestimmt Aristoteles durch ihre Fähigkeit, im Einzelfall die Angemessenheit der strikten Anwendung der Gesetze und der durch sie reglementierten Ausübung der Strafgerechtigkeit zu reflektieren und das allgemeine Gesetz zu Gunsten der Angemessenheit im Hinblick auf den Einzelfall zu berichtigen (vgl. ebd.). Die Notwendigkeit einer Korrektur der ausgleichenden Gerechtigkeit sieht Aristoteles durch die Allgemeinheit des Gesetzes begründet, insofern dieses den Besonderheiten des Einzelfalls niemals gänzlich gerecht wird. Wenn die Anwendung des Gesetzes in Form der Ausübung von Strafgerechtigkeit zu Unrecht führen würde, sieht Aristoteles die Korrektur der ausgleichenden Gerechtigkeit als ethisch gerechtfertigt an: "Dies ist also das Wesen des Billigen, eine Berichtigung des Gesetzes zu sein, insofern dieses wegen seiner Allgemeinheit eine Lücke aufweist « (ebd.: 189, 1137b).

Das Konzept der Billigkeit wurde im 20. Jh. insbesondere durch den Neokantianer Hermann Cohen aufgegriffen, der in der »Ethik des reinen Willens « die Billigkeit als ein zentrales Konzept der Ethik bestimmt (vgl. Cohen 1904: 587). Cohen hebt hervor, dass die Reflexion über den Verzicht auf ausgleichende Gerechtigkeit zur Sicherung des Fortbestandes zwischenmenschlicher Interaktion jenseits eines unendlichen Regresses der Schuldvergeltung die besondere Fähigkeit und Aufgabe der Ethik gegenüber dem Recht sei. Er fordert weiterhin, über Aristoteles hinausgehend, eine umfassende Etablierung des Konzepts der Billigkeit im Recht:

»Die Billigkeit muss dem strikten Recht angegliedert werden, als eine homogene Art des Rechts; damit sie das Problem des Besonderen, das an sich unlösbare, für die Rechtsfindung immer mehr zugänglich mache« (ebd.).

Im Gegensatz zur Amnestie, die durch eine Entscheidung eines souveränen politischen Machthabers erlassen werden kann und auf die der Angeklagte grundsätzlich dezidiert kein Recht hat, würde die Etablierung der epieikeia als ethische Gerechtigkeit im Sinne Cohens die Möglichkeit einer Einforderung der Strafmilderung oder des Straferlasses ermöglichen, und zwar, wie es insbesondere im Konzept der asymmetrischen Bestrafung zur Korruptionsbekämpfung gefordert wurde, sex anter (siehe Zitat Lambsdorff/Nell 2007). Das bedeutet, die Möglichkeit der Strafmilderung würde als ein systematisches Recht auf Einzelfallprüfung für Hinweisgeber in Aussicht gestellt.

Die systematische Aussicht auf Strafmilderung oder Straferlass bietet einen Schlüssel zur Aufdeckung der Ordnung der Geheimhaltung und eine Möglichkeit der Fundierung von bereits vorhandenen Ansätzen des Integritätsmanagements, die auf Systeme für anonyme Hinweisgeber oder non-retaliation policies für Hin- 
weisgeber setzen. Mit diesem ethischen Gegengewicht zur Strafgerechtigkeit würde eine Tür zu Netzwerken der Korruption geöffnet, indem die Ungewissheit über das Risiko der Aufdeckung in Netzwerken der Korruption erhöht würde. Außerdem diente ein solches Gegengewicht dazu, die weiteren Effekte der Strafgerechtigkeit, die in diesem Artikel nicht in Frage gestellt werden, überhaupt erst freizusetzen.

\section{Ungewissheit als Kriterium für ethische Entscheidungen}

Die schwierige Situation von Risikoanalysen, die im Hinblick auf die Prävention von Korruption mit einem viel zu kleinen Potenzial der Aufdeckung von Korruption rechnen müssen, ${ }^{2}$ könnte durch die Anreizsetzung und systematische Ansetzung von asymmetrischer Bestrafung für Hinweisgeber verbessert, die Ungewissheit in der Ordnung der Geheimhaltung der Korruption deutlich erhöht werden.

Auch aus ethischer Sicht lässt sich das Kriterium der Ungewissheit in seinem Potenzial für ethische Entscheidungen ausweisen. So hat der französische Sozialphänomenologe Emmanuel Levinas (1974) die Ungewissheit als entscheidendes Kriterium für das Treffen ethischer Entscheidungen ausgewiesen und es in Zusammenhang mit dem oben bereits genannten Begriff der Billigkeit gebracht. Er greift dieses Konzept im Rahmen seiner Verantwortungstheorie auf, indem Verantwortung für ihn das Abwägen zwischen dem Anspruch des stets durch Fremdheit und Unberechenbarkeit gekennzeichneten individuellen Anderen und den allgemeinen normativen Ansprüchen aller anderen >Anderen` in Form der Gesellschaft, der damit verbundenen Institution des Gesetzes und normativen Ordnung, die er übergreifend als den >Dritten< bezeichnet (vgl. Levinas 1974, 2002; Bedorf 2003), impliziert. Verantwortung liegt für Levinas in der ethischen Entscheidung zwischen dem singulären und durch Ungewissheit gekennzeichneten Anspruch des Anderen und dem `Dritten <, d.h. der institutionalisierten gesellschaftlichen Instanz allgemeiner Normen und Gesetze. Die Möglichkeit der Korrektur und Aussetzung ausgleichender Gerechtigkeit hebt er dabei im Anschluss an Cohen und Aristoteles als eine besondere Fähigkeit der Ethik hervor und bezeichnet sie durch den französischen Begriff der equité: Billigkeit (vgl. Levinas 1974: 210).

\section{Konklusion}

Die von Levinas in Form eines Differenzprinzips vorgestellte Unberechenbarkeit und Ungewissheit des >Anderen $>$ hat nicht nur im Hinblick auf eine ethische Legitimierung der asymmetrischen Bestrafung Relevanz. Vielmehr setzt eine systematische Erhöhung der Ungewissheit in geheimen Ordnungen der Korruption den Hebel der Reflexion über die Berechenbarkeit des korrupten Interaktionspartners

2 Das Ausmaß von Korruption z.B. in Deutschland lässt sich nur schwer schätzen, die Dunkelziffer wird für sehr hoch gehalten (vgl. Handelsblatt vom 11.11.2004, »Die bestechliche Republik «). 
sowohl an einer Kosten-Nutzen-Rechnung auf der Ebene rationaler Erwartung als auch an der Vertrauenswürdigkeit auf der Ebene der emotionalen Dimension der interpersonalen Beziehung an. Sie setzt die Unberechenbarkeit zukünftiger Folgen und Risiken eines korrupten Deals als Faktor für eine Entscheidung gegen Korruption ein. Damit ließe sich durch die asymmetrische Bestrafung für Einzelfälle von Hinweisgebern die systemische Ebene des geheimen Netzwerkes erodieren.

Jedoch erforderte ein Umdenken im Hinblick auf die Verfassung der Gerechtigkeit eine ethische Reflexion und Hinterfragung der im öffentlichen Raum propagierten Axiome der Moral, der Praxis der Schuldzuweisung und moralischen Verurteilung Einzelner und dem damit verbundenen öffentlichen Ruf nach ausgleichender Gerechtigkeit, die die für Korruption charakteristische Ordnung der Geheimhaltung zementieren und nicht unterlaufen. Dies impliziert einen reflektierten Umgang mit der Tatsache, dass Korruption ein komplexes Netzwerkphänomen ist, das insbesondere in schweren Fällen internationaler Korruptionsnetzwerke nicht durch die Forderung ausgleichender Gerechtigkeit für einzelne Verantwortliche, sondern nur durch einen systemisch ausgerichteten Ansatz der Erodierung der Geheimhaltung angemessen adressiert und überhaupt zur weiteren rechtlichen Ahndung freigelegt werden kann. Die Etablierung eines Begriffs ethischer Gerechtigkeit für Einzelfallentscheidungen über die Strafmilderung für Whistleblower sowie die Förderung von Unternehmens- und Organisationskulturen, die anstelle öffentlicher Repräsentation auf ein internes Integritätsmanagement setzen und anstelle der massiven Androhung von Sanktionen ein ausbalanciertes System der Fehlerreflexion und -behebung über alle Hierarchiestufen hinweg ausarbeiten, eröffnete eine ethisch angemessene Perspektive für die Korruptionsbekämpfung und -prävention. Allerdings lässt sich eine solche Kultur der ausbalancierten ethischen Reflexion angesichts von bereits bestehender und zu verhindernder Korruption nur unter der Maßgabe umsetzen, dass sie sich nicht gleichzeitig zu Zwecken der öffentlichen Repräsentation, des damit zumeist verbundenen Gedankens des Marketings oder/und zum Zwecke der Verschleierung nutzen ließe.

\section{Literaturverzeichnis}

Alloa, E. (2017): Transparenz als Zensur, Süddeutsche Zeitung vom 27.06.2017.

Aristoteles (2006): Nikomachische Ethik, Hamburg: Rowohlt.

Baecker, D. (2000): Ämter, Themen und Kontakte: Zur Form der Politik im Netzwerk der Gesellschaft, in: Priddat, B. (Hrsg.): Der bewegte Staat, Marburg: Metropolis.

Beck, L./ Nagel, V. (2012): Korruption aus ökonomischer Perspektive, in: Graeff, P./Grieger, J. (Hrsg.): Was ist Korruption? Baden-Baden: Nomos.

Bedorf, T. (2003): Dimensionen des Dritten. Sozialphilosophische Modelle zwischen Ethischem und Politischem, München: Wilhelm Fink.

Boissevain, J. (1974): Friends of Friends: Networks, Manipulators, and Coalitions, New York: Blackwell Publishers.

Bluhm, H./Fischer, K. (Hrsg.) (2002): Sichtbarkeit und Unsichtbarkeit der Macht. Theorien politischer Korruption, Baden-Baden: Nomos.

Cohen, H. (1904): Ethik des reinen Willens, Berlin: Bruno Cassirer. 
Doe, J. (2016): Das Manifest des John Doe, Süddeutsche Zeitung vom 6. Mai 2016. Link: http://www.sueddeutsche.de/wirtschaft/panama-papers-das-manifest-von-john-doe-1.298 2442 (zuletzt abgerufen am 30.06.2017).

Emmenegger, S. (2016): Das Yates-Memorandum: Verschärfung im amerikanischen Unternehmensstrafrecht, AJP 2016, 1045-1055.

Engels, J. I. (2014): Die Geschichte der Korruption: Von der Frühen Neuzeit bis ins 20. Jahrhundert, Berlin: S. Fischer.

Fischer-Lescano, A. (2015): Internationalrechtliche Regulierung des Whistleblowing. Anpassungsbedarf im deutschen Recht, Juristisches Kurzgutachten im Auftrag des DGB, Zentrum für Europäische Rechtspolitik (ZERP), Universität Bremen.

Foucault, M. (1977): Überwachen und Strafen, Frankfurt: Suhrkamp.

Heidbrink, L./Rauen, V. (2016): Warum Wirtschaftsphilosophie? Eine kontroverse Auseinandersetzung, in: Enkelmann, W. D./Priddat, B. P. (Hrsg.): Was ist? Wirtschaftsphilosophische Erkundungen, Bd. 3, Marburg: Metropolis, 183-207.

Heimann, R. (2015): Die Frage nach Gerechtigkeit: Platons Politeia I und die Gerechtigkeitstheorien von Aristoteles, Hobbes und Nietzsche, Berlin: Duncker\&Humblot.

Hénaff, M. (2010): The Price of Truth. Gift, Money and Philosophy, Stanford: Stanford University Press.

Hénaff, M. (2011): Die pervertierte Gabe. Tugend, Heuchelei und Nihilismus. Zu einer Anthropologie der Korruption, in: Lettre international 93, 54-56.

Homann, K. (1997): Unternehmensethik und Korruption, in: Zeitschrift für betriebswirtschaftliche Forschung (zfbf) 49, 187-209.

IDW Prüfungsstandard (2011): Grundsätze ordnungsmäßiger Prüfung von Compliance Management Systemen (IDW PS 980), Düsseldorf: IDW Verlag.

Klitgaard, R. (1991): Gifts and Bribes, in: Zeckhauser, R. J. (Hrsg.): Strategy and Choice, Cambridge/Mass: MIT Press.

Lambsdorff, J. Graf/ Teksoz, S. U. (2002): Corrupt Relational Contracting, Diskussionsbeiträge aus dem Volkswirtschaftlichen Seminar der Universität Göttingen, Beitrag Nr. 113.

Lambsdorff, J. Graf/Nell, M. (2007): Fighting Corruption with Asymmetric Penalties and Leniency, Göttingen: Center for European, Governance and Economic Development Research Discussion Paper 59, University of Goettingen, Department of Economics.

Levinas, E. (2011 [1974]): Jenseits des Seins oder anders als Sein geschieht, Freiburg: Karl Alber.

Levinas, E. (2002 [1962]): Totalität und Unendlichkeit. Versuch über die Exteriorität, Freiburg: Karl Alber.

Lübbe, H. (2001): Ich entschuldige mich - Das neue politische Bußritual, Berlin: Siedler.

Luhmann, N. (1997): Die Gesellschaft der Gesellschaft, Frankfurt a. M.: Suhrkamp.

Neckel, S. (1995): Der unmoralische Tausch. Eine Soziologie der Käuflichkeit, in: Enzensberger, H. M. (Hrsg.): Kursbuch 120, 9-16, Berlin: Rowohlt.

Nietzsche, F. (1999 [1887]): Zur Genealogie der Moral, in: Colli, G. von/Montinari, M. (Hrsg.): Jenseits von Gut und Böse, Zur Genealogie der Moral, kritische Studienausgabe, Bd. 5, München: de Gruyter.

Paine, L. S. (1994): Managing for Organizational Integrity, in: Harvard Business Review, Vol.: 72/No. 2, 106-117.

Priddat, B. P. (2005): Schwarze Löcher der Verantwortung: Korruption - die negative Variante von Public-Private-Partnership, in: Jansen, S. A./Priddat, B. P. (Hrsg.): Korruption. Unaufgeklärter Kapitalismus - Multidisziplinäre Perspektiven zu Funktionen und Folgen der Korruption, Wiesbaden: VS Verlag für Sozialwissenschaften, 85-103.

Priddat, B. P./Schmid, M. (Hrsg.), (2011): Korruption als Ordnung zweiter Art, Wiesbaden: VS Verlag für Sozialwissenschaften.

Rauen, V. (2015): Die Zeitlichkeit des Verzeihens. Zur Ethik der Urteilsenthaltung, München: Wilhelm Fink. 
Rauen, V. (2016): Corruption: Uncovering the Price of Normative Morality and the Value of Ethics, in: Wolf, S./Graeff, P. (Hrsg.): Special Issue on Ethical Implications of Corruption, German Law Journal, Vol. 17/No. 1, 101-118.

Ricour, P. (2004 [2000]): Gedächtnis, Geschichte, Vergessen, München: Wilhelm Fink.

Rose-Ackerman, S. (1999): Corruption and Government. Causes, Consequences and Reform, Cambridge: Cambridge University Press.

Sandel, M. ( 2013 [2009]): Gerechtigkeit. Wie wir das Richtige tun, Berlin: Ullstein.

Sandel, M. (2012): What Money Can't Buy: The Moral Limits of Markets, New York: Ingram International Inc.

Schmid, M. (2011): Mafia, Warlords, Terror, Korruption: Systeme rationaler Besitzsicherung, in: Priddat, B. P./Schmid, M. (Hrsg.): Korruption als Ordnung zweiter Art, Wiesbaden: VS Verlag für Sozialwissenschaften, 99-139.

Simmel, G. (1989): Philosophie des Geldes, Frankfurt a. M.: Suhrkamp.

Stenner, C. (2011): Die Funktion von Korruption. Über die Besonderheit informaler Institutionen in der japanischen Gesellschaft, in: Priddat, B./Schmid, M. (Hrsg.): Korruption als Ordnung zweiter Art, Wiesbaden: VS Verlag für Sozialwissenschaften, 163-190.

Stessl, A. (2012): Interdisziplinäre Strategien im Compliance Management, in: Graeff, P./Grieger, J. (Hrsg.): Was ist Korruption? Baden-Baden: Nomos.

Streck, B. (1995): Geben und Nehmen. Oder die Korruption in den Tiefen der Menschheit, 120 Kursbuch: Korruption, 1, 5, Berlin: Rowohlt.

Waldenfels, B. (2006): Schattenrisse der Moral, Frankfurt a. M.: Suhrkamp.

Walzer, M. (1983): Spheres of Justice, New York: Basic Books.

Wieland, J. (2005): Die Governance der Korruption, in: Jansen, S. A./Priddat, B. P. (Hrsg.), Korruption. Unaufgeklärter Kapitalismus - Multidisziplinäre Perspektiven zu Funktionen und Folgen der Korruption, Wiesbaden: VS Verlag für Sozialwissenschaften, 43-63.

Wolf, S. (2009): Die Siemens-Korruptionsaffäre - ein Überblick, in: Graeff, P./Schröder, K./ Wolf, S. (Hrsg.): Der Korruptionsfall Siemens. Analysen und praxisnahe Folgerungen des wissenschaftlichen Arbeitskreises von Transparency International Deutschland, BadenBaden: Nomos, 9-17.

Yates, S. (2015): Individual Accountability for Corporate Wrongdoing, Memorandum, US Department of Justice. 